\title{
HAEMORRHAGE AND COAGULATION A PRACTICAL APPROACH
}

\author{
Gordon Lyons* \\ Consultant Obstetric Anaesthetist \\ St James' University Hospital, Leeds LS9 7TF, United Kingdom \\ *Corresponding author E-mail: glyons@blocked.org.uk
}

Key words: Obstetric, Hemorrhage. Maternal Mortality

\section{Induction}

In the United Kingdom a national hospital service is freely available to all citizens. Apart from a small number who deliver at home, virtually all of the 700,000 women who deliver each year do so in public hospitals. Private maternity units are unknown outside London. The District Hospitals where most deliver often have blood banks, coagulation laboratories and intensive care facilities available on site. This may have some significance for the way that haemorrhage is managed in the UK.

\section{Maternal Deaths from Haemorrhage}

A unique feature of practice in the UK is that data collection is well developed. Mortality statistics are available from the early part of the last century, and have been collected without a break since the last world war ${ }^{1}$. They show that 50 years ago, around 40 women a year died from haemorrhage compared with 3 to 4 now, and this pattern has been established for more than 25 years. The introduction of routine ultrasound scanning and elective Caesarean section have reduced deaths from placenta praevia, but abruption and uterine atony deaths have also declined. This suggests that the factors responsible for the improvement in mortality are general in nature, and common to all causes of obstetric haemorrhage. Consequently, haemorrhage is now the $7^{\text {th }}$ most important cause of maternal death in the UK, less important than cardiac disease and suicide, and even amniotic fluid embolism².

\section{Pathophysiology of haemorrhage}

Haemorrhage is accompanied by an increasing sympathetic response, diverting circulation away from the periphery and the gut. An important contributing factor to maternal death is failure to appreciate the severity of the situation. Because the women are mostly young and fit, and are able to compensate well, worrying hypotension implies a significant loss [3].

\begin{tabular}{|l|l|}
\hline Volume lost & Clinical symptoms \\
\hline $\begin{array}{l}\text { Up to 15\% } \\
\text { blood volume } \\
\text { around } 900 \mathrm{~mL}\end{array}$ & $\begin{array}{l}\text { mild increase in pulse rate } \\
\text { normal blood pressure }\end{array}$ \\
\hline $\begin{array}{l}15 \% \text { to } 30 \% \\
\text { blood volume } \\
1000 \text { to } \\
1500 \mathrm{~mL}\end{array}$ & $\begin{array}{l}\text { heart rate } 110-130 \mathrm{bpm} \text { urine } \\
\text { output declines subtle central } \\
\text { nervous system changes }\end{array}$ \\
\hline $\begin{array}{l}\text { 30\% to 40\% } \\
\text { blood volume } \\
\text { around 2000mL }\end{array}$ & $\begin{array}{l}\text { heart rate 120 - 160 bpm fall in } \\
\text { systolic blood pressure } \\
\text { cold peripheries, pale skin, } \\
\text { change in mental status }\end{array}$ \\
\hline $\begin{array}{l}\text { More than 40\% } \\
\text { blood volume } \\
\text { In excess of } \\
\text { 2500ml }\end{array}$ & $\begin{array}{l}\text { very fast heart rate Systolic } \\
\text { blood pressure less than } \\
\text { 80mmHg } \\
\text { no palpable peripheral pulses } \\
\text { urine output small or absent } \\
\text { confusion or unconsciousness } \\
\text { (immediately life threatening) }\end{array}$ \\
\hline
\end{tabular}

A new recommendation to try and improve detection is the introduction of Modified Obstetric Early Warning Scores (MEOWS) [2].

Rising systemic vascular resistance and failing perfusion generate acid metabolites in the tissue groups deprived of circulation. A falling $\mathrm{pH}$ has a negative impact on myocardial function, which only serves to generate further acidosis. This will be offset initially, by the increase in catecholamine production. Volume replacement and oxygen delivery on the appropriate scale is 
required to reverse the trend of shut down, acidosis and failing myocardium. Because obstetric losses can be brisk, a particular efficient administration system is required. Monitoring $\mathrm{pH}$ can be a useful measure of whether replacement is keeping up with the loss. Short-term inotrope administration can help overcome the negative effects of acidosis ${ }^{3}$.

\section{Risk Factors for Haemorrhage}

Age, parity and Caesarean delivery are associated with increased risk of bleeding. In the UK, the mean maternal age is now 29, with 27 being the mean age for a first baby ${ }^{4}$. The risk of Caesarean delivery increases with advancing age. An elective operation has three times the mortality of a vaginal delivery, and placenta praevia, abruptio placentae and uterine tony are responsible for most instances of significant bleeding. Coagulation failure complicates a small number of cases.

\section{Placenta praevia}

The traditional grading for praevia reflects the risk attached to vaginal delivery. An alternative grading based on the position of the placenta relative to a uterine incision, and the presence of a uterine scar, is suggested. A low anterior placenta attached to a uterine scar can cause torrential bleeding and successive maternal mortality reports have repeatedly warned of the danger ${ }^{1,2}$. Despite this there seems to be reluctance amongst anaesthetists to regard this as core knowledge. Women continue to die from a haemorrhage that can be anticipated. New recommendations are that ultrasound imaging is used to identify the site of the placenta in all women who have a uterine scar and where abnormal placentation is suspected, magnetic resonance imaging is indicated ${ }^{2}$. Blood loss may be reduced with regional anaesthesia, and increasingly this is being used ${ }^{6-8}$.

\section{Placental abruption}

Even a small central abruption can cause fetal demise. Bleeding may be concealed or revealed. A consumptive coagulopathy can complicate. Because abruption can be a feature of preeclampsia and HELLP syndrome, associated renal and hepatic dysfunction contribute to a syndrome of multi organ failure. Even appropriate management of haemorrhage in these circumstances can complicate further, as the ability to accommodate colloid loading is limited, and organ support may be required.

\section{Uterine atony}

Long, obstructed labours predispose to failure of uterine contraction. Medical management includes oxytocics, ergot derivatives, prostaglandin analogues and manual compression. Examination should first exclude undetected tears and lacerations.

\section{Surgical management}

Temporary control of bleeding might be possible from direct compression of the aorta. The B-Lynch compression suture and ligation of the internal iliac arteries are steps to consider, proceeding to hysterectomy if control is not obtained. Interventional radiologists can offer embolisation if temporary control allows transfer to an imaging suite.

\section{Coagulation failure}

Intravascular volume replacement may give rise to a dilutional coagulopathy, as factors V and VIII, together with fibrinogen and platelets are washed out. Massive haemorrhage can trigger a consumptive coagulopathy, and also create the circumstances required for dilutional coagulopathy. Conditions such as abruption and amniotic fluid embolism are associated with a coagulopathy that might contribute to the haemorrhage. For all these reasons, a practical approach does not seek to distinguish between dilutional and consumptive causes of coagulopathy.

\section{Coagulation monitoring}

The traditional coagulation screen is useful provided the response time is quick enough for the results to have some meaning. An alternative is to use a point of care coagulation monitor, and the greatest experience has been obtained with the thromboelastograph, which will give a response in ten to twenty minutes ${ }^{9}$. Use in cardiac surgery has allowed a reduction in the use of expensive blood components. Each system has strengths and weaknesses.

\section{Practical approach}

Haemorrhage is a potential problem in every obstetric unit, and all units should have a protocol for its management. More recent recommendations 
suggest that the protocol is tested regularly by fire drill style practices.

Success requires the mobilisation of the necessary resources, additional staff, the transfusion service and the coagulation laboratory.

Short large bore cannulae, which give the greatest flow, attached to a rapid infusion system are necessary to keep up with loss. Simple alternatives to expensive infusion devices are described. Warmed fluids, and pressure channels for arterial and central venous monitoring should also be available. Direct arterial monitoring is invaluable and can be achieved without electronic devices, whilst central venous pressure is associated with greater risk and provides only fine tuning.

Target values are an important way of directing activity. When the mean arterial pressure is below $80 \mathrm{mmHg}$, infusion should be brisk. Input should be managed to keep the $\mathrm{pH}$ above 7.2. A falling $\mathrm{pH}$ suggests that losses are not being adequately replaced. The target haematocrit might be set at $30 \%$, this threshold triggering blood replacement.

Serial measurement of blood count, blood gases, and coagulation screen are needed. Serum calcium, potassium and lactate can also be useful.

Finally, continuing support should be available in a high dependency or intensive care unit.

Lessons from successive maternal mortality reports suggest that the commonest error is underestimating the severity of blood loss. Setting clinical targets to guide management and monitoring intensively are one way to avoid this mistake.

\section{References}

1 Confidential Enquiries into Maternal and Child Health. The $6^{\text {th }}$ Report on Confidential Enquiries into Maternal Deaths in the United Kingdom; Why Mothers Die 2000-2002. RCOG Press, London 2004

2 Confidential Enquiries into Maternal and Child Health. The $7^{\text {th }}$ Report on Confidential Enquiries into Maternal Deaths in the United Kingdom; Saving Mothers' Lives 2003-2005. CEMACH, London 2007

3 Pargger M, Schneider M. Major haemorrhage. In: Russell I F, Lyons G (eds) Clinical Problems in Obstetric Anaesthesia. Chapman and Hall, London1997, pp 33-46
4 Thomas J, Paranjothy S. Royal College of Obstetricians and Gynaecologists. Clinical Effectiveness Support Unit. The National Sentinel Caesarean Section Audit Report. RCOG Press, London 2001, pp 24-32

$5 \quad$ Hall M, Bewley S (1999) Maternal mortality and mode of delivery. Lancet 354: 776

6 Frederiksen M C, Glassenberg R, Stika C S. Placenta praevia: a 22year analysis. American Journal of Obstetrics and Gynecology 1999; 180: 1432

7 Parekh N, Husaini S W, Russell I F. Caesarean section for placenta praevia: a retrospective study of anaesthetic management. British Journal of Anaesthesia 2000; 84: 725

8 Hong J-Y, Jee Y-S, Yoon S M K. Comparison of general and epidural anesthesia in elective cesarean section for placenta praevia totalis: maternal hemodynamics, blood loss and neonatal outcome. International Journal of Obstetric Anesthesia 2003; 12: 12-6

9 Gorton H, Lyons G. Is it time to invest in a thromboelastograph? International Journal of Obstetric Anesthesia 1999; 8: 171-178 